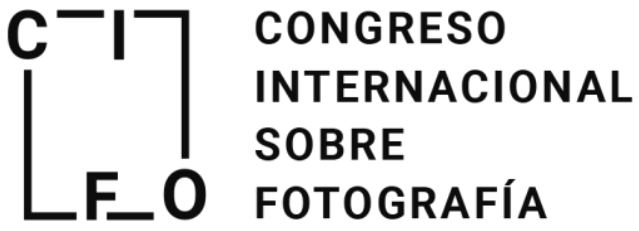

Congreso Internacional sobre Fotografia

UPV, 5 y 6 octubre 2017

Doi: http://dx.doi.org/10.4995/CIFo17.2017.6769

ISBN: 978-84-9048-604-7

\section{La fotografía y el territorio como herramienta de aprendizaje. Dos ejemplos prácticos}

\author{
Javier Fernández Pérez de Lis \\ javierfernandezperezdelis@gmail.com
}

\begin{abstract}
The relation between photography and territory during the formative process is the main topic in this researching. Focusing on the territory and enviroment as it depicts into the construction of selfknowkedge and personal develpement during secondary studies. Using the photography as the main tool that brings the opportunity to think over those ideas, as well as the way we construct images and the different possibilities. The researching is developed under the contemporary art context and tools.
\end{abstract}

Keywords: Photography, Territory, Selfknowledge, City, Urbanity, Rurality, Develpement, Learning, Analogue, History, Publishing

\footnotetext{
Resumen

La investigación llevada a cabo intenta establecer una relación entre la fotografía y el territorio dentro de la educación secundaria. De este modo, trabajamos con el concepto de territorio como parte fundamental de la formación personal, la autoestima y el conocimiento personal, siendo la fotografía la herramienta que nos permite reflexionar sobre estas ideas, así como sobre la propia construcción de las imágenes y sus diferentes posibilidades. La investigación se desarrolla de un modo práctico introduciendo herramientas y referencias del arte contemporáneo.
}

Palabras clave: Fotografia, Territorio, Autoconocimiento, Ciudad, Urbanidad, Ruralidad, Desarrollo, Aprendizaje, Analogía, Historia, Publicación 


\section{Introducción}

La siguiente investigación pretende abordar el uso de la fotografía como herramienta dentro de la educación secundaria para el auto-conocimiento y para un análisis crítico del desarrollo de nuestro entorno.

Se trata de mostrar como a través de la fotografía podemos trabajar con estudiantes de secundaria la necesidad de conocer e involucrarse con lo que nos rodea. Como a través de la imagen, podemos replantearnos la forma en que nos desenvolvemos y como construimos las ciudades y el territorio.

Ademas, es importante recalcar que lo hacemos a través de las herramientas del arte contemporáneo, de sus formas de hacer y comprender la realidad. Por lo que existe una parte procedimental que tiene mucha importancia para ubicar la investigación dentro de un contexto concreto.

Se trata, por lo tanto, de un trabajo de carácter cualitativo, donde el alumnado genera unos resultados acorde a las prácticas llevadas a cabo con ellos, además de su propia construcción personal o sus conocimientos previos. Esto, genera unos resultados amplios y distintos según el número de participantes y su naturaleza.

Se trata de un proyecto efectuado en dos centros con características muy distintas, donde proponemos la misma práctica a grupos de estudiantes con necesidades, comportamientos y naturalezas distintas. Estos son un Centro de Educación Especial, y un Instituto de Enseñanza Secundaria.

En este sentido, poder trabajar con la fotografía les ofrece la posibilidad de descubrirse, de descubrir otra parte de su propio mundo, de su propio espacio, es lo que sitúa el trabajo práctico de la investigación en un lugar de experimentación y/o liberación para el alumnado que ha participado.

\section{Marco Teórico. Investigación/empírica vs trabajo profesionalizante.}

Las diferentes vertientes del proyecto de investigación generan esta posibilidad de situar el trabajo en dos parámetros distintos.

Por un lado, el trabajo intenta mostrar una investigación sobre el uso de una herramienta en el aula, así como diferentes conceptos sobre los que desarrollar el trabajo. (urbanidad, ruralidad, entorno, formación,...) Y por otro lado, realizamos esta investigación de forma real dentro de dos centros educativos, en dos aulas distintas, pudiendo incluir esta unidad dentro del curriculum de un centro educativo, teniendo entonces, las características de un trabajo profesionalizante.

En cualquier caso, enmarcamos este trabajo dentro de una investigación-acción.

\section{Estado de la cuestión.}

Esta investigación surge con la premisa de introducir el trabajo con la fotografía y el territorio dentro del aula. La posibilidad de que el alumnado pueda trabajar con una herramienta como la fotografía de modo individual ofrece grandes posibilidades de experimentar, fomentando la capacidad visual y el pensamiento crítico. Al trabajar con fotografía analógica nos obliga además a reflexionar sobre la construcción de las imágenes ya que el proceso no es inmediato como en la fotografía digital que les puede ser mas familiar, al tiempo que la propuesta nos invita a pensar sobre el territorio.

La misma base metodológica que desarrollamos en estos centros se ha usado en diferentes proyectos artísticos, cito como ejemplo, los presentados en el contexto de la Fotobienal de Vigo;en 1992, el trabajo del grupo TAFOS (Talleres de Fotografía Social) en Perú, y en la edición del año 2000, el trabajo realizado en Belo Horizonte No Olho da Rúa de Julian Germain. 
Otros ejemplos pueden ser el trabajo de Francis Flurin y Halea Isabelle Kala, Augen-Blick (2015, http://www.haleaisabellekala.com/Projects/Augen-Blick) donde para retratar la realidad de los refugiados en Berlín, entregan unas cámaras desechables a cinco participantes. O dentro del sector audiovisual, la película Todos vos sodes Capitáns, de Olvier Laxe, donde juega con esta misma idea de entregarle a terceros la cámara, aportando de este modo una mirada nueva, incluso naif.

De igual modo, proyectos como Fotografía En Curso ( CGAI), Territorio e Identidad, Experiencia formativa a través de la fotografía y la participación de la juventud, (2009, Sánchez Montalbán, Francisco J. , Benítez Castejón, $\mathrm{M}^{\mathrm{a}}$ José e Alonso Bisquert, Francisca) entre otros, son ejemplos de la inclusión de la fotografía en el aula, o del uso de la imagen para fomentar la participación social y una educación mas activa.

\section{Objetivos}

La investigación se llevó a cabo en dos centros distintos de la ciudad de Vigo. Por un lado, un instituto de secundaria del casco urbano, donde se les entregaron cámaras desechables para trabajar con las premisas propuestas durante aproximadamente siete días.

En segundo lugar, un Centro de Educación Especial, en un área semi-rural, con un grupo de estudiantes de reprografía. A los que les entregué cámaras instantáneas cedidas por Fuji, con las que trabajaron durante el mismo periodo de tiempo, siete días.

Los objetivos del trabajo eran poder insertar la fotografía en el aula, y de algún modo, hacer reflexionar sobre las imágenes fotográficas, las que ya existen y las que hacemos, de forma que el proceso de fotografiar sea algo más que apretar un botón. Dicho de otro modo, que el hecho fotográfico adquiera un significado.

Desarrollo los objetivos del trabajo en tres ejes fundamentales:

\section{La fotografía}

La fotografía dentro del campo del arte contemporánea es el medio en el que trabajo de forma habitual y el que me interesa para realizar la investigación.

Introducir la fotografía dentro del aula puede ayudarnos a establecer una relación mas adecuada y personal en diferentes conceptos, y nos acerca de un modo mas abierto a los objetivos que perseguimos para este trabajo. Es decir, la fotografía puede funcionar como herramienta facilitadora para acercarnos otros propósitos dentro de un grupo de trabajo. ( herramienta de expresión, herramienta de crítica, etc..)

Considero que la educación dentro de lo visual es fundamental para un mejor entendimiento del mundo contemporáneo, de nuestra historia, nuestro presente, así como una preparación adecuada para el futuro próximo del alumnado.

También, pienso que puede ayudarnos a inculcar el pensamiento crítico, el debate y el trabajo colaborativo por medio de los procesos de toma fotográfica o edición y selección de los trabajos.

Como decía en el primer párrafo, vinculo directamente el mundo de la fotografía con el arte contemporáneo, ya que la considero un instrumento idóneo y preciso para la transmisión y expresión de ideas y conocimiento en un trabajos personales.

Son numerosos los artistas que usando la fotografía reflexionan sobre el espacio y el territorio. Diferentes tendencias dentro del arte y/o la fotografía focalizan el modo en que se trata el tema. Artistas como Bleda y Rosa, que desde la memoria y la historia dialogan con el territorio,; Xavier Ribas, desde una perspectiva política 


\section{La fotografía y el territorio como herramienta de aprendizaje.Dos ejemplos prácticos}

de construcción social, analiza los modos de distribución topográfica; o el trabajo de fotógrafos americanos como Alex Soth, o clásicos como Stephen Shore, usando el paisaje desde una perspectiva de lo cotidiano y recorriendo de este modo un territorio descrito por sus propias imágenes.

Dentro de este proceso de investigación, usamos la fotografía analógica por ofrecer unos parámetros que la fotografía digital , a pesar de ser mas accesible para el alumnado, no puede ofrecer.

Me interesa suprimir esa inmediatez de la cámara digital, o por lo menos, esa sensación de "infinito" que puede dar el repetir una fotografía hasta tener los resultados adecuados.

Dentro de nuestro experimento, la fotografía analógica juega un papel fundamental para obligarnos a pensar, a detenernos antes de hacer una toma, a reflexionar sobre el espacio, la situación y el momento. De esta forma, intento que ese instante se valore de un modo distinto, pues en el mejor de los casos, un participante podrá sacar 20 fotografías, lo cual le limita y le obliga a pensar cada una de las imágenes que quiere tomar.

Ocupa también, la fotografía, el lugar de ser un vehículo para la expresión de chicas y chicos que no la usan de manera habitual. El grupo del CEE ( Centro de Educación Especial), usaron la fotografía como medio para expresar y enseñar su entorno mas íntimo. Habitaciones, familias, etc.. Obteniendo de la fotografía un complemento y un cómplice para mostrar las cosas que de otra forma no pueden hacerlo.

Dentro de la enseñanza en el mundo de la fotografía existen ciertas publicaciones de referencia, como pueden ser los libros de Michael Langford, ( La fotografía paso a paso, editado en inglés en 1979) o los propios manuales publicados por Ansel Adams, The negative, The print, The Camera. Aunque pienso que para este proyecto de carácter docente, usé otras referencias alguna de las experiencias reseñadas en el libro The Photographers Playbook ( Aperture, 2015), donde le editor, Jason Fulford, reúne mas de trescientas experiencias docentes de diferentes artistas y fotógrafos, y/o ejemplos de tareas para hacer con grupos de estudiantes.

Por supuesto, el material escrito y tratado por Allan Sekula, sobre la semiótica y la capacidad de la fotografía para significar. Así como la relación directa que hace Sekula entre fotografía y la política. Pienso que esos dos pilares entablan perfectamente con los patrones del trabajo realizado. Refiriéndonos no solo a la fotografía en si, si no que la herramienta, el uso, la toma, y los significados que pueden adquirir para los participantes.

\section{El Territorio}

El territorio como elemento caracterizador de una persona, y por lo tanto, concepto indispensable para el desarrollo personal del alumnado. Mediante el trabajo con el territorio podemos generar contenidos que acentúan la formación para la igualdad, la reflexión y el conocimiento personal.

Comprender el territorio nos ayuda a definir nuestra situación en el mundo, a construir un mapa de nuestro crecimiento y relación con lo fuera.

El arte trabaja con el territorio desde prácticas como el situacionismo, ( Guy Debord, Théorie de la Derive, in Les Lévres nues, n.9, noviembre 1956, Bruxelles), trabajos dentro del land art como Robert Smithson (https://gd1studio2011.files.wordpress.com/2011/09/smithson-monuments-of-passaic.pdf)

O desde el trabajo mas local y lo contemporáneo con el territorio como la propuesta de Carme Nogueira ( A Trama Rururbana, Nos camiños,..) o mas reciente el colectivo Montenoso ( http://montenoso.net) que crea una comunidad para trabajar y repensar el territorio desde diferentes perspectivas. No se puede obviar el trabajo de Proxecto Terra (http://proxectoterra.coag.es) que lleva 15 años vinculado al trabajo con el entorno, con la arquitectura y el espacio de Galicia dentro del marco educativo y de formación, colaborando con diferentes centros educativos. 
Son todas estas aproximaciones al territorio desde una construcción social y desde el arte, pero existen muchos ejemplos que podríamos citar en estas líneas en relación al trabajo desarrollado en el proyecto de investigación.

Debemos plantearnos la necesidad de trabajar desde lo local, el territorio, el espacio. De este modo, desde lo "local", podemos llegar a lo "internacional". Por eso concibo de una forma necesaria la educación y la reflexión de como construimos el territorio y nuestra propia comunidad.

Desde esta práctica recogemos la necesidad de construir un lugar mejor, de reflexionar sobre como se ordena la ciudad y el territorio y nos puede ayudar a expresarnos y opinar sobre los procesos de aprendizaje personal que nos afectan en relación al espacio.

\section{La pedagogía}

La mayoría de los ejemplos propuestos anteriormente llevan implícita una teoría pedagógica. Una propuesta para trabajar desde la construcción educativa, y que se nutre de pensamiento vinculado al mundo de la pedagogía directamente.

Dentro de este campo, me interesa el trabajo desarrollado por pedagogos de carácter socialista, como pueden ser algunas de las experiencias llevadas a cabo en los años treinta como el Método Freinet, o el trabajo por proyectos vinculados a pedagogías libres como Montessori. Muchas de estas tendencias heredadas de las teorías y practicas de escuela progresiva propuesta por Dewey y la escuela como laboratorio.

“La educación está reprimiendo los talentos y habilidades de muchos estudiantes y está matando su motivación para aprender. ” Ken Robinson, (Robinson, Punset and Aronica, 2014)

Basándome en la palabras de Paulo Freire, “Enseñar no es transferir conocimiento, si no crear las posibilidades para su propia producción o construcción. ", el ejercicio de mi experimento se centra en el propio trabajo y producción con el grupo a trabajar, y conforme la pedagogía de pensamiento crítico en la que se basan las ideas de Freire, considero que la experimentación con el alumnado, introduciéndoles una variable a su rutina y permitiéndoles tomar la iniciativa sobre lo que tienen que realizar y sus resultados. Esto produce una emoción que les permite aprender, generar pensamiento crítico al mismo tiempo que se disfruta del trabajo.

Por eso, llevar a cabo este proyecto dentro de aulas de educación reglada tiene una motivación especial para mi, pues me interesa interferir en el sistema idealizado en una jerarquía de aprendizaje, que sitúa las materias creativas en la parte mas baja de importancia, excluyendo casi por completo las posibilidades para crear conocimiento de las materias en expresión plástica, sin fomentar el uso de experiencias, las emociones, la visión y el pensamiento para aprender a aprender.

Mi idea es intentar fomentar la experiencia como proceso de formación y crecimiento, utilizar esto como parte del proceso de auto-construcción de la personalidad dentro del desarrollo del alumnado.

\section{Desarrollo de la innovación}

\section{Materiales y métodos.}

O Meu Territorio. La actividad propuesta se llama de este modo ya que la idea es trabajar desde la fotografía interpretando y reconociendo el espacio propio de los participantes en la actividad.

Comenzamos con un test inicial para valorar el punto de partida, que conocimientos previos tiene le grupo para poder tomar en el proyecto hacia unos resultados de la investigación. 


\section{La fotografía y el territorio como herramienta de aprendizaje.Dos ejemplos prácticos}

Posteriormente, muestro una proyección con nociones básicas de fotografía, mostrando como ésta puede suponer una herramienta para la expresión y reconocimiento del espacio, sea desde un punto de vista personal o físico.

Trabajamos en el grupo con diferentes cámaras, y enseñamos como funcionan. Mientras explico la actividad a realizar en los siguientes siete días, les entrego las cámaras que emplearán.

En sesiones siguientes, recogemos el material y trabajamos con las fotografías ya reveladas. Seleccionando y editando, primero en pequeños grupos, y luego en puesta en común con el resto de compañeros, para entre todos, en gran grupo, hacer la selección final de las fotografías que conforman el trabajo de cada grupo.

Con este resultado, editamos un libro o publicación donde se muestran los resultados y fotografías. Es importante tener una sesión donde el alumnado pueda ver la finalización del proyecto, de forma que pueda darle valor al trabajo realizado.

Mi proyecto consta de dos volúmenes, uno por centro educativo con el que trabajé, siendo ambos diferentes a pesar de que los parámetros solicitados a los participantes es el mismo. Esta variación reside en la individualidad de cada uno de los participantes, las características personales y las del grupo,

\section{Recursos}

Los recursos que empleamos durante la actividad son aquellos que disponemos en el aula, así como los que dispongo yo para la realización del proyecto. Desde cámaras fotográficas antiguas, cámaras obscuras, a libros y fotografías impresas o ordenador y proyector para las sesiones en el aula. Para el trabajo de campo, contamos con cámaras fotográficas desechables en un caso y cámaras instantáneas Fuji Instax en el otro. Empleamos también diferentes recursos como la impresión digital o imprenta la finalización del proyecto en una publicación.

\section{Metodología de investigación}

El enfoque a usar en la investigación es de carácter cualitativo.

Los datos a recoger provienen del ejercicio generado con el alumnado participante, ofreciendo así una visión personal de cada una de las partes de la investigación.

La investigación se centra en estos dos grupos de alumnos, un grupo de educación especial en zona semirural y un grupo de secundaria en ámbito urbano. Por lo tanto, el grupo esta formado por personas con edades comprendidas entre los 12 y los 16 años.

Además del material recogido por los participantes, el material de la investigación se compone también del primer test inicial y de una entrevista en la última sesión, para recoger datos y opiniones de la actividad.

\section{Resultados}

Existen diferentes resultados en lo que respecta al proceso de investigación.

Por un lado, todos los propios del proceso "per se" recogidos entre los diferentes instrumentos para el análisis, como el test inicial, la entrevista final, o los que forman parte de la observación directa. 
Por otro lado, los resultados mostrados de una manera práctica en los volúmenes publicados con el trabajo realizado por los participantes del proyecto, su implicación en el trabajo, etc..

\section{Resultados por centro}

\section{A. PROYECTO 1. INSTITUTO DE EDUCACIÓN SECUNDARIA.}

A raíz de la observación directa sobre el grupo, detecto el interés generado por mi propuesta. No se si se debe a la ruptura que a mi participación en el aula supone o a un interés real polo que vamos a trabajar.

La comprobación de los test de conocimientos previos muestran algunas carencias formativas, no solo dentro de los aspectos plásticos sino dentro de conocimientos generales. Los conocimientos en fotografía son limitados. De forma general pueden localizar cierta información (inicios da fotografía en blanco e negro), pero mas de la mitad non contextualiza correctamente la época de invención ( por ejemplo) de la fotografía. Esto indica que estamos a tratar una materia con carencias a nivel formativo, pero que viendo los resultados enriquece diferentes aspectos de la formación del alumnado.

Durante las explicaciones, funcionamos de una manera democrática, donde cualquier participante pode intervenir, compartir e preguntar cualquier duda. A observación directa, el grupo se muestra activo, participando $\mathrm{y}$, aparentemente asimilando las distintas cuestiones que se plantean y resolvemos en el aula.

Disponen de una semana con los equipos fotográficos para realizar el trabajo. A pesar de ofrecerme a solventar posibles dudas durante el trabajo, no hay ninguna cuestión o problema durante este tiempo.

De forma general, se muestran cercanos y próximos, interesados por la publicación con sus trabajos. Muestran interés en continuar reflexionando sobre los diferentes aspectos trabajados durante la actividad.

\section{CONCLUSIÓNS PERSONALES}

El trabajo en un centro de secundaria urbano, resultó, en cierta medida, como algo que esperaba. Chicas y chicos que inician su adolescencia. Esto supone que al realizar una actividad extracurricular de estas características, se relajen en el aula, compartiendo y comportándose de un modo distinto de lo que harían si yo fuera su docente principal. La atención de los alumnos es buena, y los resultados, una vez vistos, reflejan algunas de las inquietudes que tienen, formados por los diferentes temas tratados como son su entorno, el propio centro de estudios, su tiempo libre, amistades, familia y futuro.

\section{B. PROXECTO II. CENTRO DE EDUCACIÓN ESPECIAL.}

Las conversaciones con la directora del centro me sitúan en lo que me voy a encontrar en el aula. Alumnado con diferentes afecciones que limitan su comportamiento.

Se trata de un grupo de nueve personas tan distintas como en el centro anterior, pero con particularidades que debemos tener en cuenta a la hora de desarrollar el proyecto.

Una vez en el aula, el interés es muy grande, la aceptación a mi presencia genera mucha ilusión en clase y aportan sus dudas y miedos a medida que se les ocurren. 
El test inicial muestra dos cosas. Por un lado, que la formación para la imagen y o la fotografía no es específica, por lo que los resultados son derivados de la propia experiencia o convivencia del alumnado con la fotografía.

Por otro lado, me demuestra que este test no es adecuado para el tipo de centro con el que estoy trabajando. Alguno de los participantes no se pude concentrar, contestan de forma inconsciente o no le gusta demostrar sus ideas en el papel. Pienso si es necesario este test, o sería mucho mas apropiado realizar este paso previo con una entrevista.

El alumnado se involucra totalmente en el aula. Comparten sus preocupaciones y miedos por trabajar con una cámara analógica e con un material que no es de ellos, pero demuestran un gran interés y expectativas para trabajar durante los siguientes días en la propuesta que les hago.

Durante una semana disponen de los equipos para realizar el trabajo. A pesar de ofrecerme a solventar sus posibles dudas durante este periodo, no hay ninguna cuestión o problema. Cuando voy a recoger el material compruebo que muchos no acabaron y/o que tuvieron mas dudas durante el proceso. Decidimos acabar el trabajo todos juntos durante un rato antes de pasar a la siguiente sesión.

Comparto dos sesionas mas con este alumnado. En ellas, muestran gran interés en compartir su trabajo conmigo. Hablamos de lo que supone esta herramienta para transmitir, expresarse y hablar de cosas de las que no suelen hablar. Me declaran su alegría por poder realizar el trabajo, y pensar su propia situación.

\section{CONCLUSIONES PERSONALES}

Tengo diferentes sentimientos y pensamientos sobre la actividad desarrollada en el Centro de Educación Especial. Los resultados plásticos conforman otro mapa diferente al realizado en el Instituto de Enseñanza Secundaria, pero que posee unas características propias, y que sin duda, se convierte en un volumen de expresividad fundamental para el grupo que realizó la actividad.

Tengo la sensación de haber aportado algún tipo de conocimiento, idea o, tal vez, de ofrecerles posibilidades de hacer cosas distintas. Desde las conversaciones mantenidas con el grupo, de sus ganas de seguir experimentando, de comprar cámaras como las usadas para seguir trabajando por su cuenta, etc.. esto me demuestra la necesidad de compartir este tipo de actividades y tiempo con grupos en centros de educación especial.

\section{Conclusiones}

Empezaba el proyecto de investigación con algunas hipótesis, preguntas que trataba de autorresponder o que pensaba cobrarían una importancia distinta.

La idea de trabajar sobre el territorio como proceso de construcción se ve un poco diluida una vez comenzada la experimentación, con el trabajo del alumnado.

En sus conversaciones demuestran otros intereses. A pesar de esto, los resultados son mapas del entorno en que cada alumna se desenvuelve. Pierde, tal vez, la idea mas universal de la palabra territorio, para acercarse al lado mas íntimo de la palabra espacio. Porque sin duda, lo que cada participante en el proyecto hizo fue señalar, o enseñar, partes de su propia vida. Su propio espacio. 
Otras cuestiones que me rondaban la cabeza durante todo el proceso fue la posible comparación entre los dos centros con los que realicé el taller. Por un lado, pensaba que sería interesante ver como afectan estas diferencias por la singularidad de cada participante, pero me doy cuenta que esta singularidad reside en cualquiera de los dos grupos. Por este motivo, decido que una comparación entre alumnos de un centro de secundaria y uno de educación especial carece de importancia o de rigor para la investigación, dejando el proceso en dos casos prácticos con los que he realizado la misma actividad. Pienso que en cualquier grupo o centro que realice este mismo trabajo, generará una construcción diferente, creando un volumen distinto en todos los casos.

La finalización del proyecto tiene características comunes. Publicamos un periódico con las imágenes o inquietudes de los miembros del grupo, pero con las características propias que identifican y personalizan cada una de las imágenes tomadas, cada uno de los trabajos realizados.

Decido, por tanto, no realizar esta comparativa detallada entre los grupos, dejando claro que ambos grupos ofrecieron su ánimo y esfuerzo de igual modo. Pero las realidades y el conocimiento de la propia herramienta es muy diferente, mucho mas común para los estudiantes de secundaria, por lo que valoro mucho la actitud del grupo de educación especial.

Esta es una de las ideas que me llevó a proponer este proyecto. Ofrecer una herramienta de expresión a un grupo de estudiantes que no tuvieran esta oportunidad tan a menudo, y que en cierto sentido, justifican la realización del proyecto.

Los volúmenes publicados son dos ejemplos claros del trabajo realizado. De la importancia de incluir la fotografía y diferentes formas de trabajar con la imagen dentro de las aulas, sea cual sea el tipo de personas que compongan ese grupo, pues se validan algunas de las cuestiones y creencias que tenía antes de comenzar el proyecto. El trabajo con la imagen facilita la capacidad de expresión, crea entorno de discusión y ayuda a desarrollar ciertas actitudes personales que pueden facilitar el desarrollo personal de los miembros del grupo. 


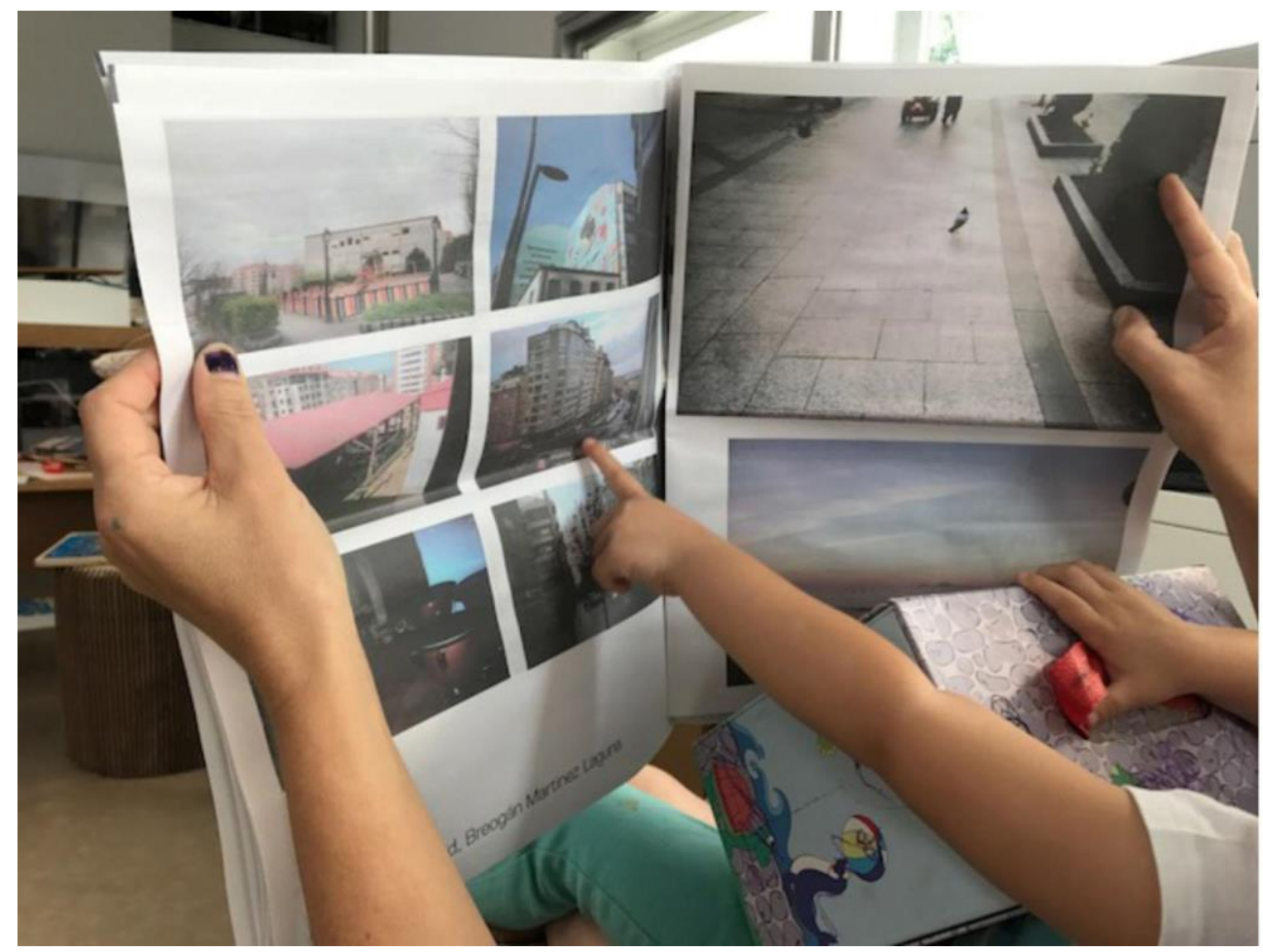

Fig. 1 Volumen 1. Instituto de Educación Secundaria

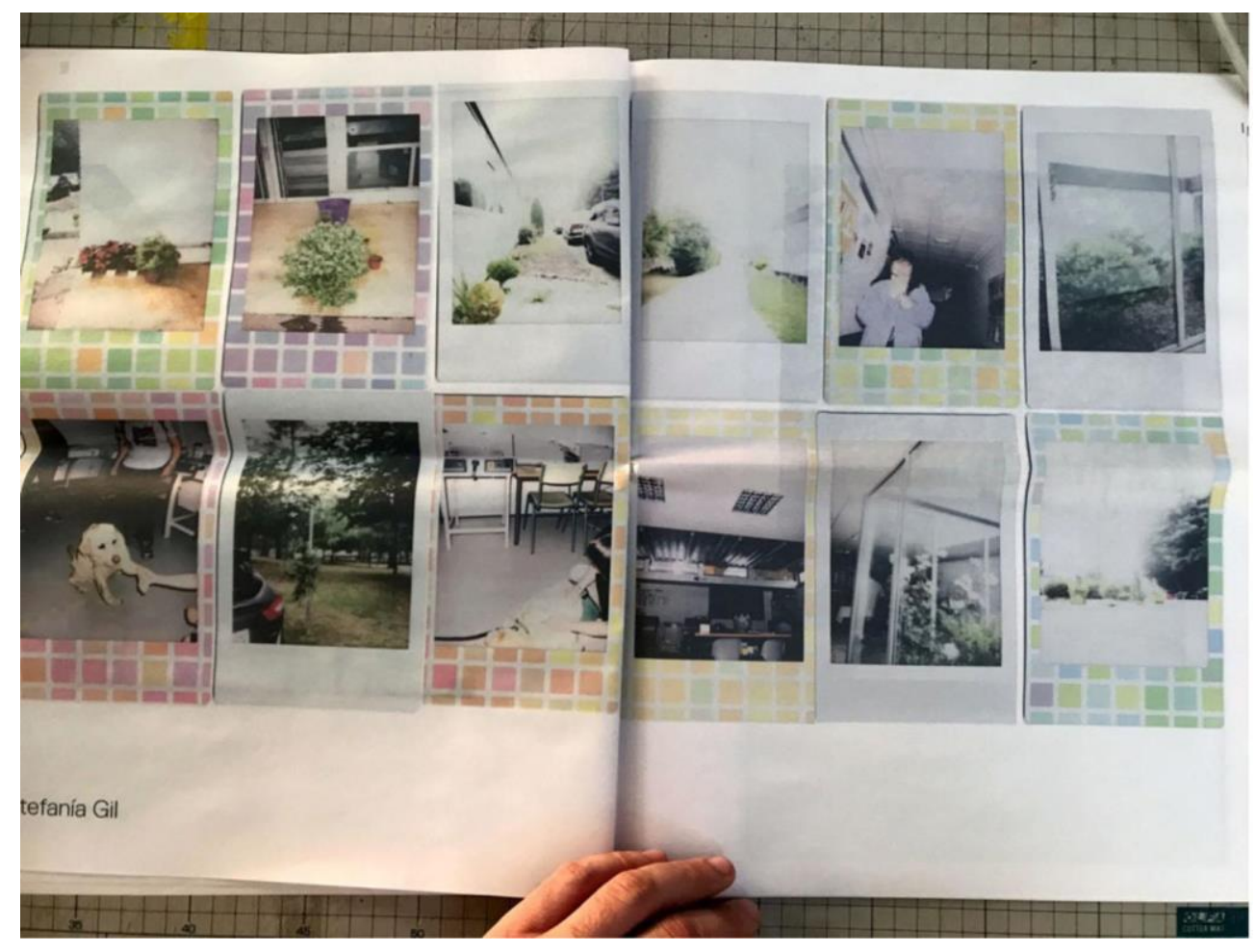

Fig. 2 VolumenII. Centro de Educación Especial 


\section{Referencias}

Libro

ANLlo, C., LOIS, M., NOGUEIRA, C. (Coord.) (2009) A trama rururbana. Documentos de Traballo., Santaigo de Compostela, CGAC, Xunta de Galicia.

FULFORD, J. (2014) The photographer's playbook, NY, Aperture.

GERMAIN, J. . EN: SENDÓN, M., SUAREZ CANAL, X.L. (com.). (2000), Fotobienal 2000. Vigo: Centro de Estudos Fotográficos,.

HUERTA, R. (2014). La mirada de los docentes hacia su ciudad:identidades urbanas y educación patrimonial. Pulso, 37. $127-147$

PARRAMÓN, R. ; CIRUGEDA, S.; AMASTÉ, CANUDAS, J. MARTÍN, J.M. SINAPSIS, PLANA, T.; SOLÉ, L.; DEMOCRACIA (2010). Catalitzadores. Arte, Educación, Territorio. Eumo Editorial y Arts Santa Mònica, Vic-Barcelona,

SEKULA, A. (2016), Photography Against the Grain: Essays and Photo Works, 1973-1983. Mack, Londres.

\section{Revista}

A RACHAS, monografía escolas. Boletin Escola Paideia. Mérida.

\section{Capítulo de un libro}

- DEBORD, G. (1956 ) «Théorie de la dérive» en Les Lèvres nues, n. 9, novembre, Bruxelles

FRANCO-VAZQUEZ, C., HUERTA, R. (2011). Educatio Siglo. La creación de una mirada urbana. La ciudad de Santiago de Compostela interpretada por el alumnado de magisterio. XXI, Vol. 29 no 2 , 229-246p.

SANCHEZ MONTALBAN, F. J.; BENITEZ CASTEJÓN, M.J.; ALONSO BISQUERT, F (2009). « Territorio e identidad. Experiencia formativa a través de la fotografía y la participación social de jóvenes». en Ciudades globales y culturas locales 2. ZAINAK. CUADERNOS DE ANTROPOLOGIA-ETNOGRAFIA. Vol. 32. EUSKO IKASKUNTZA. Páginas de 691707. Donostia,.

TAFOS (Taller de Fotografía Social) (1992) En: SENDÓN, M., SUAREZ CANAL, X.L. (com.) Fotobienal 1992. Vigo: Centro de Estudos Fotográficos.

\section{Página web}

BEDMAR, S.. La importancia del contexto en el proceso de enseñanza. Temas para la educación, revista digital para profesionales de la enseñanza. №5 2009

.https://www.feandalucia.ccoo.es/indcontei.aspx?d=3784\&s=5\&ind=178. (Consultado en Xuño 2017.)

BLEDA Y ROSA. http://www.bledayrosa.com/index.php?/proyectos/origen/(Consultado en Xuño 2017)

FLURIN F., HALEA I.K, :Augen-Blick http://www.haleaisabellekala.com/Projects/Augen-Blick. (Consultado en Xuño 2017)

MONTENOSO. Http://montenoso.gal . (Consultado en Xuño 2017)

PROXECTO TERRA . Http://www.proxectoterra.coag.es. (Consultado en Maio 2017)

WERKER MAGAZINE. http://www.werkermagazine.org/imageact. (Consultado en Maio 2017) 
La fotografía y el territorio como herramienta de aprendizaje.Dos ejemplos prácticos

\section{Películas}

Todos vos sodes capitáns... ( Dir. Oliver Laxe) Zeitun Films, 2010.

\section{Vídeo de Internet (Youtube, Vimeo...)}

PICARD GARCÍA, P. «Unha viaxe por.... . Deriva I» youtube

$<$ https://www.youtube.com/watchv=MrVTy8FRrRg $>$ [Consulta: 24 de junio de 2017] 